\title{
Erythropoietin promotes expression of survivin via STAT3 activation and reduces sensitivity to cisplatin in cervical cancer cells
}

\author{
MARÍA JOSÉ VÁZQUEZ-MELLADO ${ }^{1}$, LILIANA GUADALUPE CORTÉS-BALLINAS ${ }^{1}$, \\ IRMA CAROLINA BLANCO-FLORES ${ }^{1}$, CECILIA AGUILAR ${ }^{1}$, \\ GERARDO VÁZQUEZ-GÓMEZ ${ }^{2}$ and LETICIA ROCHA-ZAVALETA ${ }^{1}$
}

\author{
Departamentos de ${ }^{1}$ Biología Molecular y Biotecnología y ${ }^{2}$ Medicina Genómica y Toxicología Ambiental, \\ Instituto de Investigaciones Biomédicas, Universidad Nacional Autónoma de México, Circuito Escolar S/N, \\ Ciudad Universitaria, Coyoacán, Ciudad de México 04510, México
}

Received July 9, 2018; Accepted November 22, 2018

DOI: $10.3892 / o r .2018 .6890$

\begin{abstract}
Erythropoietin (Epo) is used for the treatment of cancer-associated anaemia. However, certain studies have identified that the administration of Epo mediates the acquisition of resistance to cisplatin, which is widely used to treat cervical cancer. Our group previously reported that cervical cancer cells express Epo receptor and that exogenous Epo induces cell proliferation and migration. However, the effect of Epo on cervical cancer cell death mediated by chemotherapeutic agents has not yet been evaluated. Thus, the aim of the present study was to assess the potential effect of Epo on the cytotoxic activity of cisplatin in cervical cancer cells. The effect of Epo was assessed in 3 cervical cancer-derived cell lines. It was observed that pre-incubation with Epo induced a significant reduction of cisplatin-induced apoptosis in vitro and in vivo. Incubation with Epo induced the expression and activation of the transcriptional factor signal transducer and activator of transcription 3 (STAT3), which in turn stimulated the expression and activation of the anti-apoptotic protein survivin. The cytotoxicity of cisplatin was partially restored by treating the cells with MY155, an inhibitor of survivin. Conversely, inhibition of STAT3 activation using sub-lethal doses of WP1066, completely abolished the cytoprotective effect of Epo. These observations indicated that Epo was able to hinder the cytotoxic effect of cisplatin in cervical cancer cells by activating anti-apoptotic responses regulated by STAT3.
\end{abstract}

Correspondence to: Dr Leticia Rocha-Zavaleta Departamento de Biología Molecular y Biotecnología, Instituto de Investigaciones Biomédicas, Universidad Nacional Autónoma de México, Circuito Escolar S/N, Ciudad Universitaria, Coyoacán, Ciudad de México 04510, México

E-mail:1rochaz@biomedicas.unam.mx

Key words: cervical cancer, erythropoietin, apoptosis, cisplatin, chemoresistance, signal transducer and activator of transcription 3

\section{Introduction}

Erythropoietin (Epo) is a fundamental regulator of proliferation and differentiation in erythroid progenitor cells. Human recombinant Epo has been used for the treatment of anaemia in patients with chronic conditions, including HIV infection, rheumatoid arthritis, chronic kidney disease and cancer (1). Anaemia is defined by levels of haemoglobin $<13$ and $<12 \mathrm{~g} / \mathrm{dl}$ for men and women, respectively. Anaemia is frequently detected in cancer patients: $\sim 50 \%$ of patients with solid tumours suffer from cancer-associated anaemia (2). However, the prevalence increases to $70 \%$ among patients treated with platinum-based chemotherapy (2). The use of Epo for the treatment of cancer-associated anaemia remains controversial, as there is evidence that Epo is a pleiotropic factor that exhibits proliferative and anti-apoptotic activity in various non-hematopoietic cells, including tumour cells (3).

The cytoprotective effect of Epo has been documented in various animal and in vitro models. Epo has been demonstrated to protect neuronal cells against hypoxia-induced apoptosis (4), retinal ganglional cells against high glucose-induced death (5) and kidney cells against cisplatin-induced nephrotoxicity (6). Cisplatin is an alkylating agent commonly used for the treatment of cervical cancer. Cervical cancer is the fourth most common type of cancer in women worldwide, although almost $90 \%$ of cervical cancer deaths occur in less developed countries (7). The majority of women with invasive cervical cancer suffer from chronic bleeding and they often experience nutritional deficiency, increasing the probability of anaemia development. In fact, it has been reported that in Mexico, 37\% of cervical cancer patients are already anaemic at diagnosis (8). Since these women are candidates for treatment with Epo, it is important to analyse the potential effect of Epo on cervical cancer cells.

In previous studies the authors demonstrated that cervical cancer-derived cell lines express the Epo receptor (EpoR) (9). In these cells, EpoR is activated by exogenous Epo and stimulates signalling cascades traditionally associated with cell growth [Janus kinase (JAK)/signal transducer and activator of transcription (STAT)] (9), and cell movement 
(mitogen-activated protein kinases) (10). However, the effect of Epo on cervical cancer cell death mediated by the cytotoxic activity of chemotherapeutic agents has not yet been evaluated. Thus, the aim of the present study was to assess the potential effect of Epo on the cytotoxic activity of cisplatin. It was observed that pre-incubation with Epo induced a significant reduction of cisplatin-induced apoptosis in vitro and in vivo. The inhibitory effect was mediated by the transcriptional factor STAT3, which induced not only the expression of the anti-apoptotic protein survivin, but also its activation. The cytoprotective effect of Epo was abrogated by the use of sub-lethal concentrations of WP1066, an inhibitor of STAT3. These results indicated that Epo may modify the response of cancer cells to cytotoxic drugs and that this effect is regulated by the transcriptional factor STAT3.

\section{Materials and methods}

Cells and reagents. Cervical cancer-derived HeLa (ATCC ${ }^{\circledR}$ CCL-2), SiHa (ATCC ${ }^{\circledR}$ HTB-35) and C-33A (ATCC ${ }^{\circledR}$ HTB-31) cells (American Type Culture Collection, Manassas, VA, USA) were maintained in Dulbecco's modified Eagle's medium (DMEM; Invitrogen; Thermo Fisher Scientific, Inc., Waltham, MA, USA) supplemented with $2 \%$ heat-inactivated foetal bovine serum (FBS; Invitrogen; Thermo Fisher Scientific, Inc.) and incubated at $37^{\circ} \mathrm{C}$ in a humidified atmosphere of $95 \%$ air $/ 5 \%$ $\mathrm{CO}_{2}$. Cisplatin, paclitaxel and tamoxifen were obtained from Sigma-Aldrich (Merck KGaA, Darmstadt, Germany).

Cell survival assay. The median lethal dose $\left(\mathrm{LD}_{50}\right)$ of cisplatin, paclitaxel and tamoxifen was determined by incubating HeLa, SiHa and C-33A cells with different concentrations of each compound for $24 \mathrm{~h}$. Cell viability was determined using the colorimetric 3-(4,5-dimethylthiazol-2-yl)-2,5-diphenyltetrazolium bromide (MTT) assay. To evaluate the effect of Epo on survival rates, the cells were pre-incubated with increasing concentrations of Epo for $2 \mathrm{~h}$. The cells were subsequently treated with the $\mathrm{LD}_{50}$ of each chemotherapeutic agent for $24 \mathrm{~h}$. Cell viability was evaluated by MTT assay.

Evaluation of apoptosis. Apoptosis was evaluated using the In Situ Cell Death Detection kit, Fluorescein (Roche Diagnostics GmbH, Mannheim, Germany), based on the detection of labelled DNA strand breaks [terminal deoxynucleotidyl transferase dUTP nick end labelling (TUNEL)]. Cells were fixed in $4 \%$ paraformaldehyde (in PBS, pH 7.4) for $1 \mathrm{~h}$ at room temperature. Following 2 washes with PBS, the cells were permeabilised by incubating with $0.1 \%$ Triton X-100 in $0.1 \%$ sodium citrate for $2 \mathrm{~min}$ at $4^{\circ} \mathrm{C}$. After 2 washes with PBS, the cells were labelled with the TUNEL reaction mixture for $1 \mathrm{~h}$ at $37^{\circ} \mathrm{C}$ in the dark. Cells were then mounted and analysed using a fluorescence microscope (Zeiss AG, Oberkochen, Germany). A total of 1,000 cells were counted on each slide, and the apoptosis level was expressed as the percentage of cells exhibiting immunoreactivity for the TUNEL assay. Additionally, caspase-3 activation was assessed using a Caspase-3/CPP32 Colorimetric Assay kit (BioVision, Inc., Milpitas, CA, USA), according to the manufacturer's protocol. Briefly, $1 \times 10^{6}$ cells were lysed and incubated with the $p$-nitroanilide ( $p$ NA)-labelled DEVD substrate for $2 \mathrm{~h}$ at $37^{\circ} \mathrm{C}$. Following caspase-3-mediated cleavage, free $p \mathrm{NA}$ was quantified using an ELISA plate reader (ELx-800; Bio-Tek Instruments Inc., Winooski, VT, USA) at $405 \mathrm{nM}$.

Western blot analysis. The cells were resuspended in lysis buffer (50 mM Tris-HCl, pH 7.4; 150 mM NaCl; 1 mM EDTA; $1 \%$ NP40; $0.25 \%$ sodium deoxycholate), containing $100 \mu \mathrm{l} / \mathrm{ml}$ complete protease inhibitor cocktail (Roche Diagnostics $\mathrm{GmbH}$ ) and $10 \mu \mathrm{l} / \mathrm{ml}$ phosphatase inhibitors (Sigma-Aldrich; Merck $\mathrm{KGaA}$ ). Protein concentration was determined using a DC protein assay kit (Bio-Rad Laboratories, Inc., Hercules, CA, USA). Then, $30 \mu \mathrm{g} /$ lane of total protein was resolved by $10 \%$ SDS-PAGE and transferred onto polyvinylidene fluoride (PVDF) membranes (EMD Millipore, Billerica, MA, USA). The membranes were incubated at $4^{\circ} \mathrm{C}$, overnight with the following antibodies. For the detection of human EpoR, a goat anti-human EpoR antibody (cat. no. E4644; Sigma-Aldrich; Merck KGaA), produced using a purified recombinant human Epo soluble receptor as an immunogen was used. The antibodies for the detection of JAK2 (cat. no. GTX50467), pJAK2 (cat. no. GTX61122), STAT3 (cat. no. GTX104616) and pSTAT3 (cat. no. GTX61820) were obtained from GeneTex (GeneTex, Inc., Irvine, CA, USA). Antibodies for the detection of survivin (cat. no. ab24479), and p-survivin (cat. no. ab138653) were purchased from Abcam (Abcam, Cambridge, MA, USA). As an internal control, a rabbit anti-GAPDH antibody was included (cat. no. GTX100118; GeneTex, Inc.). All primary antibodies were diluted 1:1,000. The secondary antibodies, horseradish peroxidase-conjugated anti-rabbit IgG (cat. no. GTX213110-01), and horseradish peroxidase-conjugated anti-goat IgG (cat. no. GTX228416-01) were purchased from GeneTex Inc., and they were used diluted 1:10,000. Proteins were detected by chemiluminescence using an Amersham ECL plus Western Blotting Detection System (GE Healthcare, Chicago, IL, USA).

Inhibition assays. To inhibit JAK2 phosphorylation, the cells were incubated with $10 \mu \mathrm{M}$ tyrphostin AG490 (Sigma-Aldrich; Merck KGaA) and diluted in ethanol for $24 \mathrm{~h}$. STAT3 was inhibited by incubating the cells with different concentrations $(1.09,2.19,4.38,8.77$ and $17.5 \mu \mathrm{M})$ of the STAT3 Inhibitor III, WP1066 (Santa Cruz Biotechnology, Inc., Dallas, TX, USA), and diluted in dimethyl sulfoxide (DMSO) for $24 \mathrm{~h}$. Inhibition of STAT3 phosphorylation was evaluated by western blotting. To inhibit survivin expression, the cells were incubated with different concentrations $(0.5,1.0$ and $2.0 \mathrm{nM})$ of the small molecule inhibitor YM155 (AdooQ BioScience LLC, Irvine, CA, USA), and diluted in DMSO for $24 \mathrm{~h}$. Suppression of survivin expression was evaluated by western blotting. To inhibit the expression of EpoR on the cell surface, cells were incubated with $20 \mu \mathrm{M}$ lovastatin (Sigma-Aldrich; Merck $\mathrm{KGaA}$ ) for $20 \mathrm{~h}$. Depletion of surface EpoR in membrane protein extracts was determined by western blotting.

In vivo studies. The effect of Epo on the cytotoxicity induced by cisplatin was evaluated in a xenograft model using $n u^{-} / n u^{-}$mice provided by the Animal House of the Facultad de Ciencias de la Salud, Universidad Panamericana (Mexico City, Mexico). Experimental procedures were performed in accordance with the Guidelines for the Care and Use of Laboratory Animals, and were approved by the Animal Ethics 

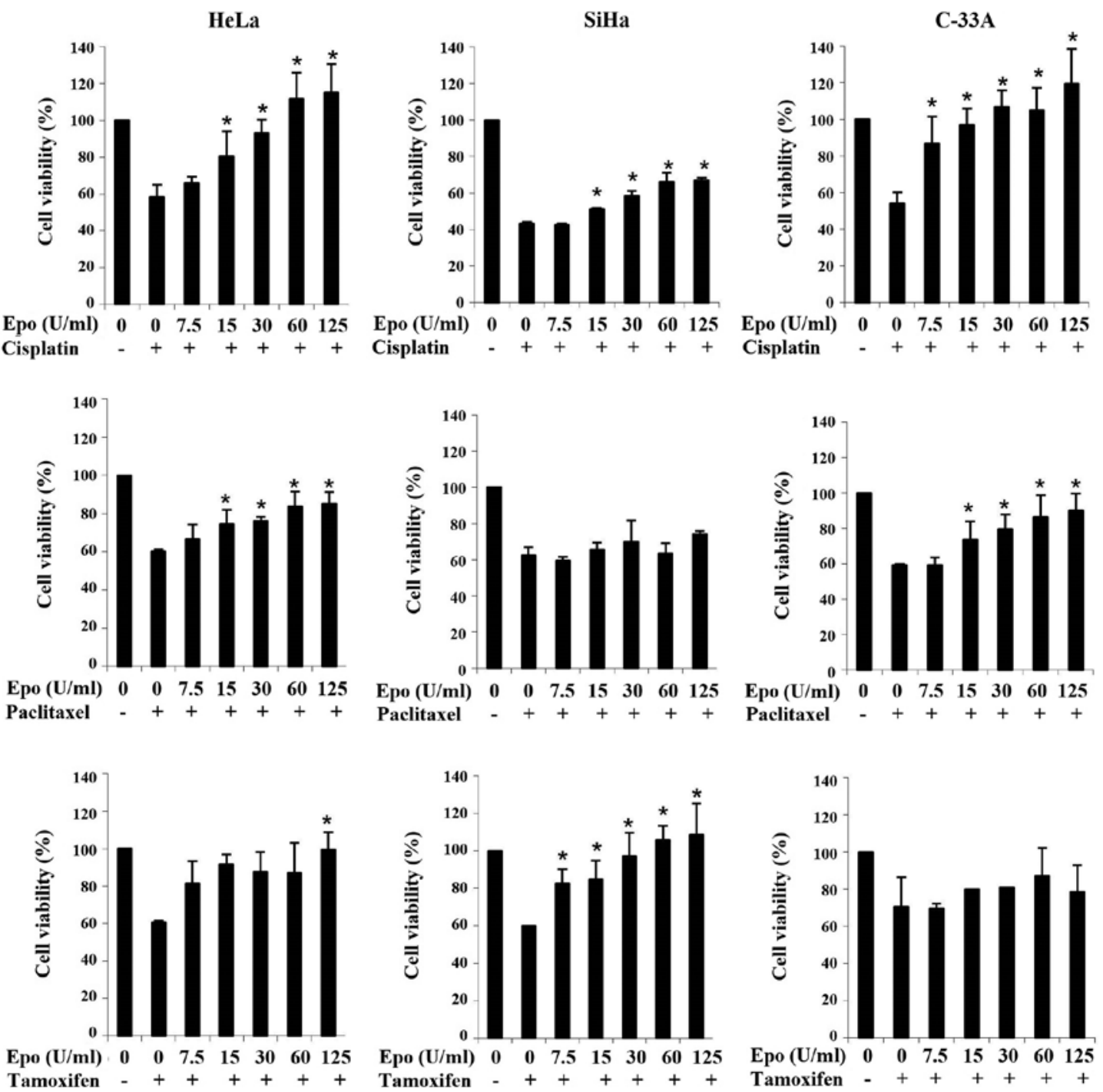

Figure 1. Epo reduces cisplatin-mediated cytotoxicity in cervical cancer cells. Cervical cancer-derived cell lines HeLa, SiHa and C-33A were pre-incubated with increasing concentrations of Epo, and then treated with the $\mathrm{LD}_{50}$ of cisplatin (upper row), paclitaxel (middle row), and tamoxifen (lower row). Cell viability was assessed by MTT assay after $24 \mathrm{~h}$. All values represent the average of 3 independent assays. Error bars indicate the standard error of the mean. ${ }^{*} \mathrm{P}<0.05$ vs. untreated controls (Tukey-Kramer test). Epo, erythropoietin; LD $_{50}$, median lethal dose; MTT, 3-(4,5-dimethylthiazol-2-yl)-2,5-diphenyltetrazolium bromide.

Committee of the Universidad Panamericana (Mexico City, Mexico). Animals were kept in a pathogen-free environment at a constant temperature of $20 \pm 2^{\circ} \mathrm{C}, 40-50 \%$ humidity, in a $12-\mathrm{h}$ light/dark cycle. They were fed ad libitum, and had free access to water. Groups of 10 female, 6-8-week-old $n u^{-} / n u^{-}$mice, with a mean weight of $17 \pm 3 \mathrm{~g}$, were implanted subcutaneously with $5 \times 10^{5} \mathrm{HeLa}$ cells in the back of the mice and monitored for tumour formation. Tumour size was measured with callipers and after 15 days the mice were treated with either $6 \mathrm{mg} / \mathrm{kg}$ cisplatin administered intraperitoneally once a week, $500 \mathrm{U} / \mathrm{kg}$ Epo by intravenous infusion twice a week, or cisplatin and Epo as indicated, for 4 weeks. To evaluate the effect of STAT3 inhibition, a group of 10 mice receiving the Epo+cisplatin regimen was treated with $20 \mathrm{mg} / \mathrm{kg}$ WP1066 intraperitoneally twice a week, the day prior to receiving Epo. For this experiment, all treatments were administered for 3 weeks. As a negative control, a group of mice received vehicle (PBS). Then, 2 days after the last inoculation mice were sacrificed by cervical dislocation after isofluorane inhalational anesthesia and the individual tumours were dissected, measured and weighed.
Statistical analysis. All results are presented as the mean \pm standard error of the mean. Differences between treatments were evaluated using one-way analysis of variance (ANOVA) test and Tukey-Kramer post hoc test with calculation of $95 \%$ confidence intervals (CIs) and P-values. $\mathrm{P}<0.05$ was considered to indicate a statistically significant difference.

\section{Results}

Epo reduces cytotoxicity mediated by cisplatin. To assess the effect of Epo on drug-induced cytotoxicity, chemotherapeutic agents exhibiting different mechanisms of action were selected. Cisplatin is an alkylating-like agent that crosslinks DNA, paclitaxel is a cytoskeletal drug that targets tubulin and tamoxifen is an oestrogen receptor antagonist. To assess the effect of Epo, HeLa, SiHa and C-33A cells were incubated with increasing concentrations of Epo and then treated with the $\mathrm{LD}_{50}$ values of cisplatin, paclitaxel and tamoxifen. As demonstrated in Fig. 1, pre-incubation with Epo resulted in a significant decrease of cisplatin-induced cell death in all 

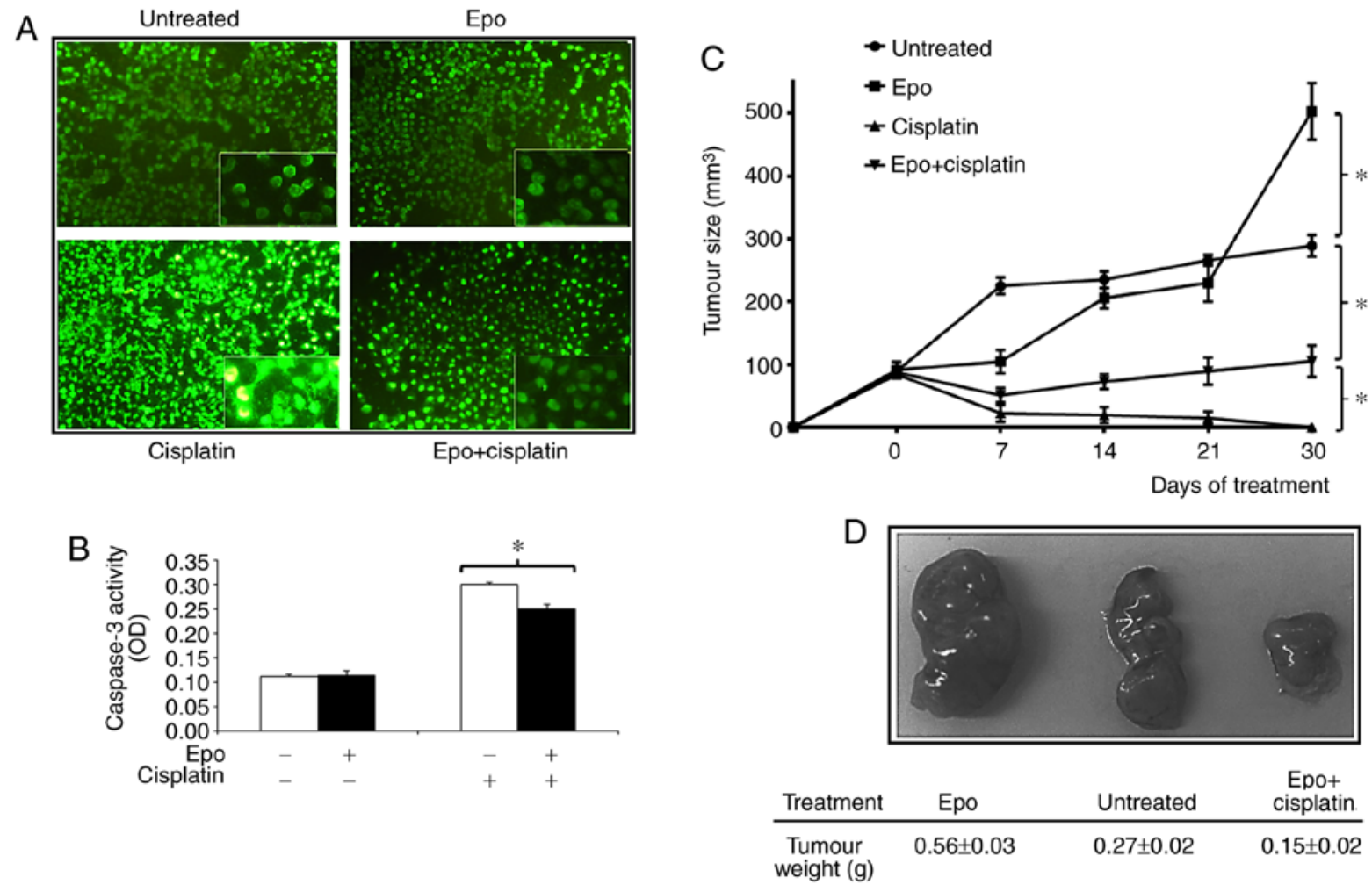

Figure 2. Epo reduces apoptosis mediated by cisplatin in vitro and in a xenograft cervical cancer model. HeLa cells were incubated with Epo or cisplatin, pre-incubated with Epo and then treated with cisplatin (Epo+cisplatin) or left untreated as a negative control. (A) Cells undergoing apoptosis were evaluated using a fluorescein-TUNEL assay. (B) Caspase-3 activity was assessed in cell lysates by a colorimetric assay. Values represent the average of 3 independent assays. Error bars indicate the standard error of the mean. ${ }^{~} \mathrm{P}<0.05$ (Tukey-Kramer test). (C) HeLa cells were implanted subcutaneously into $n u^{-} / n u^{-}$mice, and tumours were treated with Epo, cisplatin, Epo+cisplatin, or left untreated. Tumour growth was recorded every week. Data represent the means of 10 animals/experimental group. "P $<0.05$ (Tukey-Kramer test). (D) Mean weight of tumours dissected at the end of the experiment. Representative images of tumours from untreated, treated with Epo, and treated with Epo+cisplatin mice are shown. Epo, erythropoietin; TUNEL, terminal deoxynucleotidyl transferase dUTP nick end labelling.

cell lines assessed. The cytotoxic effect of paclitaxel was significantly reduced by Epo in HeLa and C-33A cell lines. Tamoxifen toxicity was significantly inhibited by Epo in HeLa and SiHa cells.

Since Epo was able to consistently inhibit the cytotoxic effect of cisplatin, which is used as first-line treatment for cervical cancer, the mechanism regulating this effect was investigated. It was first evaluated whether Epo was able to prevent cisplatin-mediated apoptosis in HeLa cells. TUNEL assay demonstrated that incubating the cells with Epo alone did not significantly modify the number of apoptotic cells (Fig. 2A). Treatment with cisplatin induced apoptosis in $59 \%$ cells. However, when the cells were pre-incubated with Epo a significant reduction of cisplatin-induced cell death was observed, with only $29 \%$ of cells undergoing apoptosis (Fig. 2A). These results were consistent with a significant decrease in active caspase- 3 detected in cells receiving Epo prior to being treated with cisplatin (Fig. 2B), indicating that Epo hindered the cisplatin-mediated apoptosis of HeLa cells. In order to evaluate the effect of Epo in vivo, HeLa cells were implanted subcutaneously into $n u^{-} / n u^{-}$mice. Tumours were treated with either cisplatin, cisplatin+Epo, Epo alone, or left untreated. As demonstrated in Fig. 2C, after 4 weeks of treatment, cisplatin induced a significant reduction in tumour size $(\mathrm{P}<0.0005)$. By contrast, when cisplatin was administered together with Epo, the tumours continued to grow. At the final time-point they were smaller than those in untreated mice $(\mathrm{P}<0.005)$, but significantly larger compared with tumours treated with cisplatin alone $(\mathrm{P}<0.05)$. As observed in a previous study (9), tumours grew significantly larger and heavier when mice were treated with Epo $(\mathrm{P}<0.0005$; Fig. 2D).

Epo induces the activation of STAT3 and the expression of survivin. The results of the present study indicated that Epo reduced the cytotoxicity induced by cisplatin. To confirm that this effect was mediated by the binding of Epo to its receptor, HeLa cells were pre-treated with lovastatin, which is a selective inhibitor of 3-hydroxy-3-methylglutaryl-coenzyme A reductase and hinders translocation of EpoR to the cell surface (11). As revealed in Fig. 3A, incubation with lovastatin induced an important reduction on the level of membrane EpoR. Depletion of surface EpoR produced a significant reduction in the protective effect of Epo (Fig. 3A). Following ligand binding, EpoR dimerization promotes the activation of the receptor-associated JAK2. Thus, to evaluate the contribution of JAK2 activity to the protective effect of Epo, cells were treated with tyrphostin AG490. As revealed in Fig. 3B, tyrphostin AG490 significantly inhibited phosphorylation of JAK2, which resulted in a significant decrease in the Epo-mediated protection against cisplatin cytotoxicity. This observation indicated that activation of a 
A
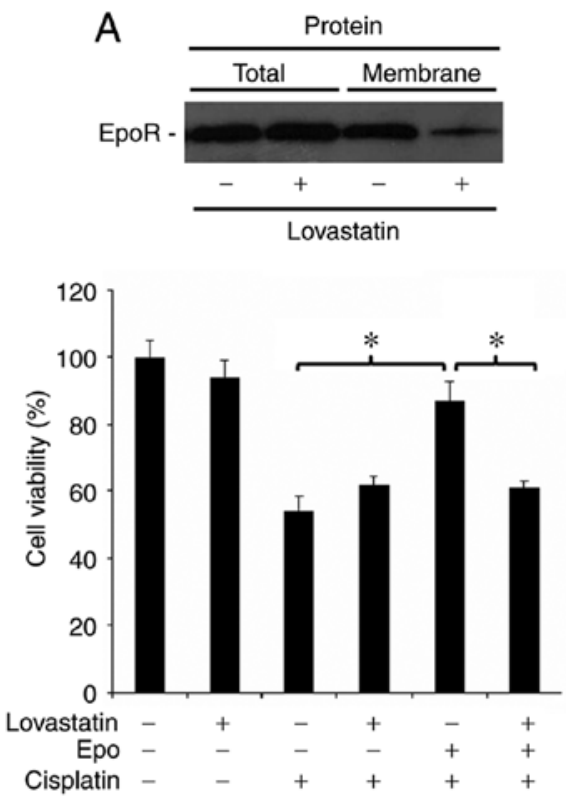

C

Epo (min)

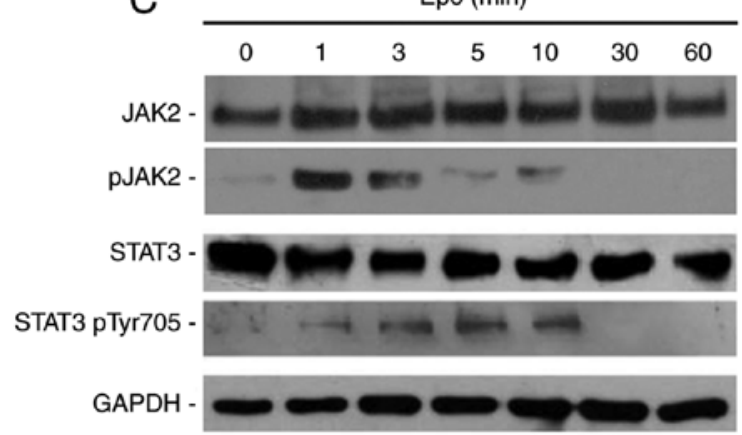

B

\begin{tabular}{|c|c|c|c|}
\hline Tyrphostin & - & - & + \\
\hline Epo & - & + & - \\
\hline pJAK2 - & & & \\
\hline
\end{tabular}

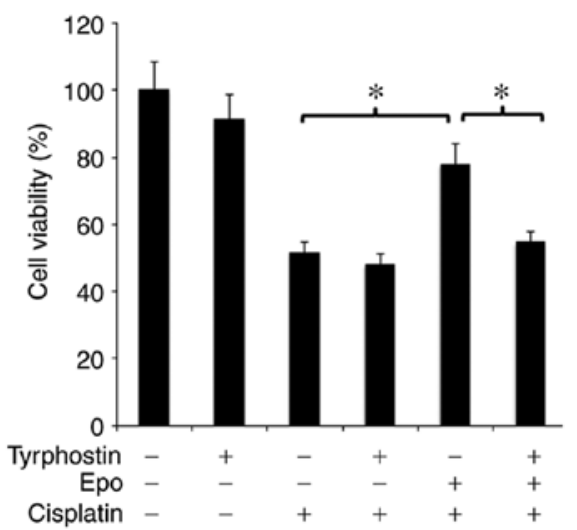

D

Epo (min)

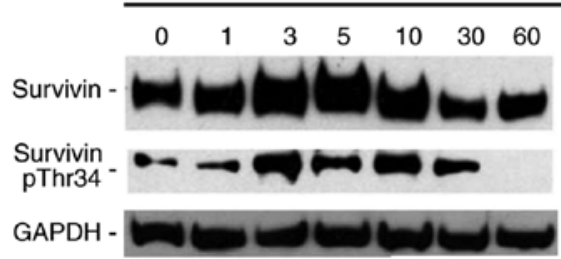

Figure 3. Epo induces cytoprotection via the activation of EpoR-associated JAK2 and stimulates the expression and activation of STAT3 and survivin. (A) HeLa cells were pre-incubated with lovastatin and then treated with Epo, cisplatin, or Epo+cisplatin. Inhibition of EpoR translocation to the cell membrane was evaluated by western blotting of total and membrane protein extracts. Cell viability was assessed by MTT assay. Each point represents the average of 3 independent determinations. Error bars indicate the standard error of the mean. ${ }^{*} \mathrm{P}<0.05$ (Tukey-Kramer test). (B) HeLa cells were pre-incubated with tyrphostin AG490 (tyrphostin) and then treated with Epo, cisplatin, or Epo+cisplatin. Inhibition of JAK2 phosphorylation was evaluated by western blotting Cell viability was measured by MTT assay. Error bars indicate the standard error of the mean. " $\mathrm{P}<0.05$ (Tukey-Kramer test). (C) HeLa cells were stimulated with $125 \mathrm{U} / \mathrm{ml}$ Epo. Phosphorylation levels of JAK2 (pJAK2) and STAT3 (STAT3 pTyr705) were evaluated at the indicated time-points by western blotting. (D) HeLa cells were stimulated with $125 \mathrm{U} / \mathrm{ml}$ Epo. Phosphorylation levels of survivin (survivin pThr34) were analysed at the indicated time-points by western blotting. GAPDH was included as a loading control. Representative images of 3 independent experiments are shown. Epo, erythropoietin; EpoR, Epo receptor; JAK2, Janus kinase 2; STAT3, signal transducer and activator of transcription 3; MTT, 3-(4,5-dimethylthiazol-2-yl)-2,5-diphenyltetrazolium bromide.

JAK2-mediated signalling pathway was involved in the protective effect of Epo. As revealed in a previous study (9), Epo not only induced the activation of the classic JAK2-STAT5 axis in cervical cancer cells, but also induced the stimulation of STAT3, a transcription factor that has been associated with the inhibition of cell death (12). Thus, in order to corroborate that Epo induces the activation of STAT3, the expression and phosphorylation of STAT3 following incubation with Epo were evaluated (Fig. 3C). As expected, Epo induced the phosphorylation of JAK 2 after 1 min of incubation. JAK 2 activation was followed by the phosphorylation of STAT3, which was evident from 3-10 min of treatment.

It is known that STAT3 is able to activate transcription of target genes associated with the control of cell death, including the anti-apoptotic protein survivin (13). Therefore, whether Epo induced the expression of survivin was subsequently assessed. As depicted in Fig. 3D, the level of survivin significantly increased following $3 \mathrm{~min}$ of treatment. Phosphorylation of the Thr34 residue has been revealed to provide survivin with stability and potentiate its anti-apoptotic activity $(14,15)$. Thus, whether Epo also induced survivin phosphorylation was determined. As observed in Fig. 3D, survivin Thr34 phosphorylation clearly increased at $3 \mathrm{~min}$ post-incubation, indicating that Epo was able to stimulate not only the expression, but also the activation of the anti-apoptotic capacity of survivin.

Cytoprotective activity of Epo is mediated by a STAT3-survivin axis. It has been demonstrated that Epo induces the activation of STAT3 (9). Thus, to address the question of whether activation of STAT3 promoted Epo-mediated cytoprotection, the cells were treated with WP1066, a potent inhibitor of STAT3 phosphorylation. Since it has been demonstrated that WP1066 is able to induce apoptosis of tumour cells (16), the effect of WP1066 on cell viability was first determined. As shown in 

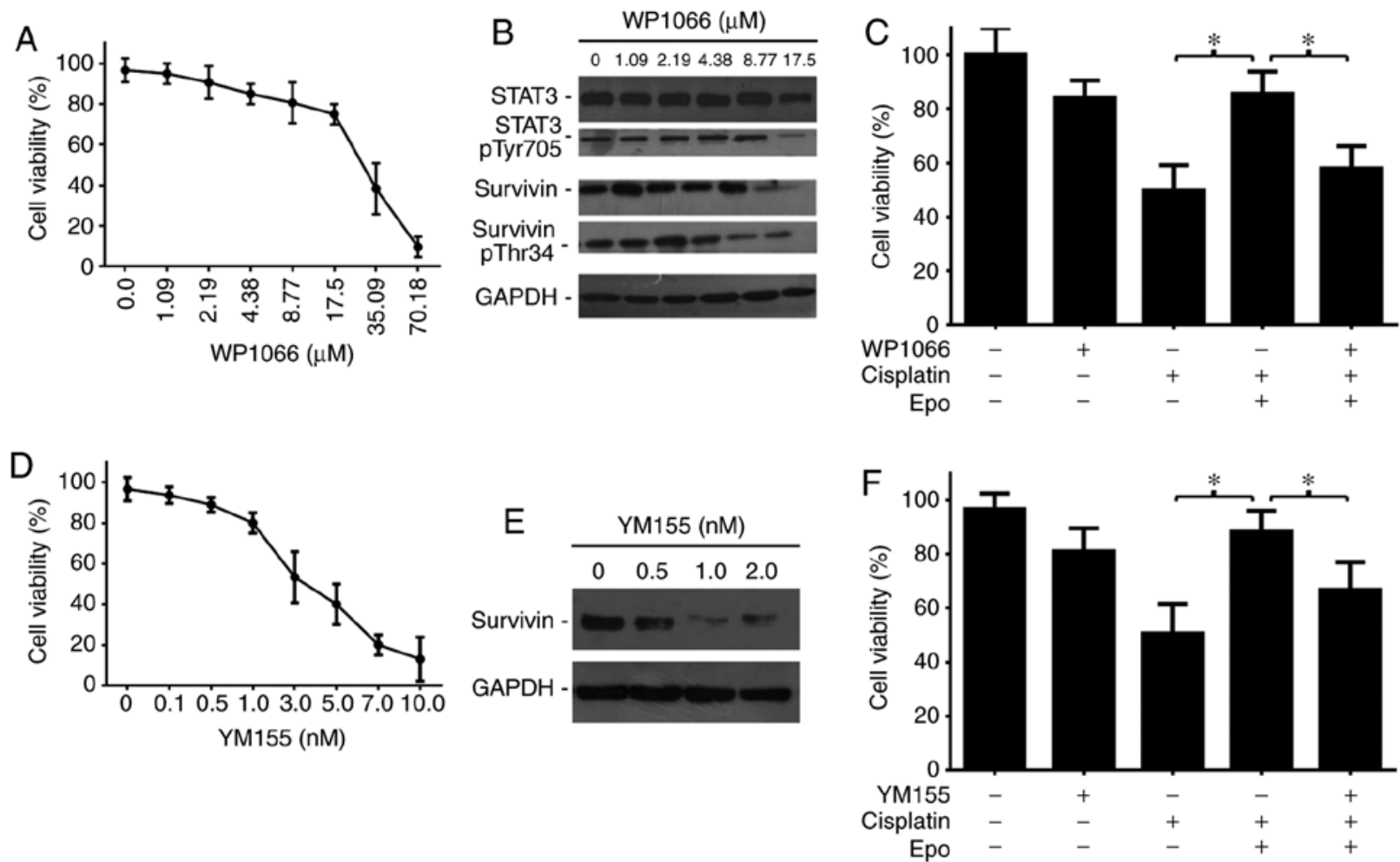

Figure 4. Susceptibility to cisplatin is restored by inhibition of STAT3 activation and partially re-established by inhibition of survivin expression. (A) HeLa cells were incubated with increasing concentrations of the STAT3 phosphorylation inhibitor WP1066. Cell viability was evaluated by MTT assay. (B) HeLa cells were incubated with the indicated concentrations of WP1066. Inhibition of STAT3 phosphorylation (STAT3 pTyr705), expression of survivin and phosphorylation of survivin (survivin pThr34), were evaluated by western blotting. GAPDH was included as a loading control. (C) HeLa cells were pre-incubated with $17.5 \mu \mathrm{M}$ WP1066 and then treated with either Epo, cisplatin, or Epo+cisplatin, and untreated cells were included as a control. Cell viability was evaluated by MTT assay. Error bars indicate the standard error of the mean. "P<0.05 (Tukey-Kramer test). (D) HeLa cells were incubated with increasing concentrations of YM155, an inhibitor of survivin gene transcription. Cell viability was evaluated by MTT assay. (E) HeLa cells were incubated with the indicated concentrations of YM155. Inhibition of survivin expression was evaluated by western blotting. GAPDH was included as a loading control. (F) HeLa cells were pre-incubated with $1 \mathrm{nM}$ YM155 and then treated with either Epo, cisplatin, or Epo+cisplatin, and untreated cells were included as a control. Cell viability was evaluated by MTT assay. In all cases values represent the average of 3 independent assays. Error bars indicate the standard error of the mean. "P $<0.05$ (Tukey-Kramer test). Epo, erythropoietin; STAT3, signal transducer and activator of transcription 3; MTT, 3-(4,5-dimethylthiazol-2-yl)-2,5-diphenyltetrazolium bromide.

Fig. $4 \mathrm{~A}$, cell viability was reduced by $25 \%$ when the cells were incubated with $17.5 \mu \mathrm{M}$ WP1066, and higher concentrations of WP1066 produced a decrease of $>50 \%$ in cell viability. Thus, to assess the effect of WP1066 on STAT3 inhibition, the cells were incubated with various concentrations of WP1066, with $17.5 \mu \mathrm{M}$ as the maximum. As revealed in Fig. $4 \mathrm{~B}$, incubation with $17.5 \mu \mathrm{M}$ WP1066 produced a significant reduction in STAT3 activation. Since the expression of survivin is regulated by STAT3, the effect of transcriptional factor inhibition on survivin expression and survivin Thr34 phosphorylation was evaluated. As expected, WP1066-mediated inactivation of STAT3 resulted in a decrease in the expression and activation of survivin. (Fig. 4B). Next, whether inhibition of STAT3 activation can alter the cytoprotective effect of Epo was investigated. The cells were first incubated with WP1066 and then exposed to Epo and cisplatin. As demonstrated in Fig. 4C, pre-incubation with WP1066 completely restored the cytotoxicity of cisplatin in the presence of Epo.

The results of the present study demonstrated that Epo stimulated survivin expression. Since survivin is a recognized anti-apoptotic factor, the participation of survivin in Epo-mediated cytoprotection was evaluated. The small molecule inhibitor YM155, which binds the survivin promoter, suppressing transactivation of the gene (17), was used. To determine the potential effect of YM155 on cell viability, the cells were incubated with increasing concentrations of YM155. Concentrations $\geq 3 \mathrm{nM}$ produced a highly toxic effect (Fig. 4D). Thus, the effect of $0.5,1.0$ and $2.0 \mathrm{nM} \mathrm{YM} 155$ on survivin expression was assessed. As demonstrated in Fig. 4E, a significant reduction of survivin was observed following incubation with $1 \mathrm{nM}$ YM155. This concentration was subsequently used to evaluate the involvement of survivin in the cytoprotective effect of Epo. The cells were incubated with YM155 prior to treatment with Epo+cisplatin, and the results are revealed in Fig. 4F. Although pre-incubation with YM155 reduced the cytoprotective effect of Epo, unlike inhibition of STAT3, interruption of survivin expression was unable to completely re-establish the cytotoxic effect of cisplatin.

The results of the present study indicated that STAT3 activation is a key regulator of Epo-induced cytoprotection. Thus, to examine the potential effect of the inhibition of STAT3 on Epo-induced cytoprotection in vivo, a xenograft model of cervical cancer using HeLa cells was established. Tumours were treated with cisplatin alone, with cisplatin+Epo, or pre-treated with WP1066 and then treated with cisplatin+Epo. Our previous results, demonstrated that administration of cisplatin completely eradicated tumours after 4 weeks of treatment. Since the present experiment aimed to compare 

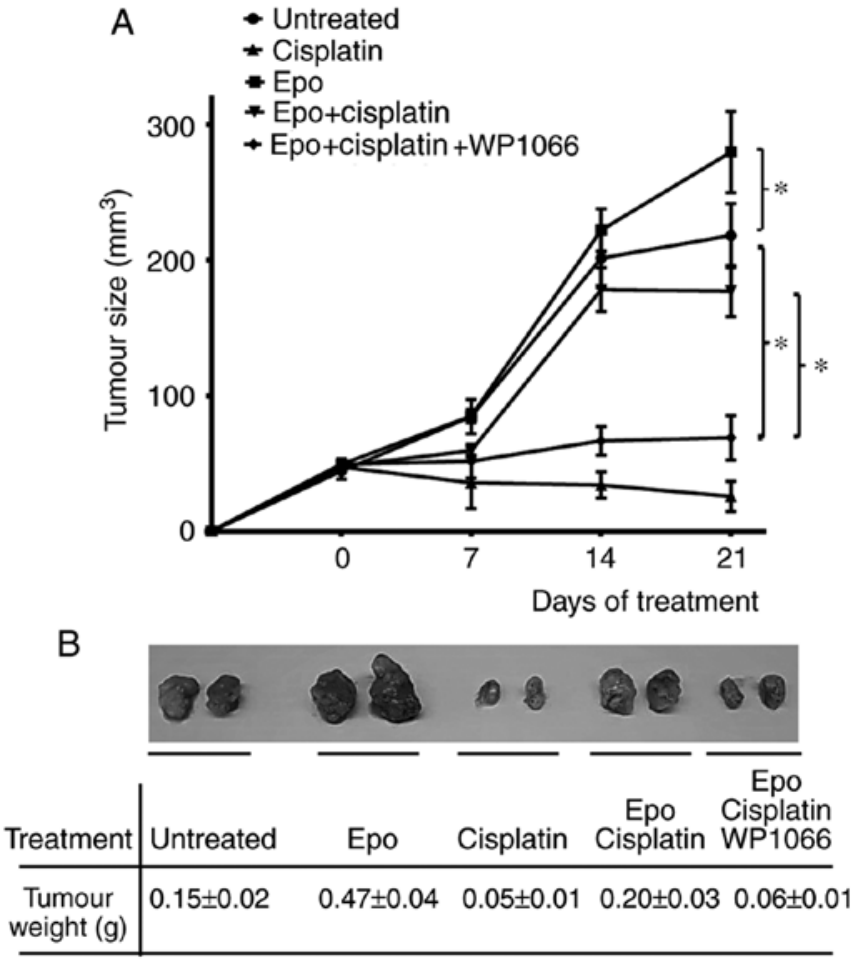

Figure 5. Inhibition of STAT3 phosphorylation restores the cytotoxic effect of cisplatin in the presence of Epo in vivo. HeLa cells were implanted subcutaneously into $n u^{-} / n u^{-}$mice, tumours were treated with Epo, cisplatin, Epo+cisplatin, Epo+cisplatin+inhibitor of STAT3 phosphorylation (WP1066 Epo+cisplatin+WP1066) or left untreated. Tumour growth was recorded every week. (A) Data represent the means of 10 animals/experimental group. ${ }^{*} \mathrm{P}<0.05$ (Tukey-Kramer test). (B) Mean weight of tumours dissected at day 21. Representative images of tumours from untreated, treated with Epo, with cisplatin, with Epo+cisplatin and with Epo+cisplatin+WP1066 mice are shown. Epo, erythropoietin; STAT3, signal transducer and activator of transcription 3 .

the effect of WP1066 on tumour size, all treatments for this experiment were carried out for 3 weeks only. The results are presented in Fig. 5.

As previously observed, administration of Epo accelerated the growth of the tumours, whereas treatment with cisplatin significantly reduced their size. As anticipated, the cytotoxic effect of cisplatin was significantly inhibited by Epo (Fig. 5A). However, the toxic effect of cisplatin was significantly increased following treatment with WP1066 (Fig. 5A). By day 21, tumours from mice treated with cisplatin+Epo were 4-fold heavier than tumours from mice receiving cisplatin alone (Fig. 5B). Conversely, tumours from mice pre-treated with WP1066 were of the same weight as those from cisplatin-treated mice (Fig. 5B), indicating that inhibition of STAT3 re-established the cytotoxicity of the drug. Collectively, these results convincingly indicated that activation of STAT3 drives the cytoprotective effect of Epo.

\section{Discussion}

Cisplatin-based chemotherapy is the established treatment for patients with cervical cancer. Thus, the acquisition of resistance to cisplatin has a negative effect on the outcome of treatment. The present study presented experimental evidence demonstrating that Epo induces resistance to cisplatin-mediated death in cervical cancer cells. The findings are in accord with previous studies revealing that administration of exogenous Epo mediated acquisition of resistance to cisplatin in glioblastoma (18) and non-small cell lung carcinoma cells (19).

A number of molecular mechanisms leading to cisplatin resistance have been described in cervical cancer cells (20). Among them, inactivation of the apoptosis pathway appears to be critical, since the anticancer effect of cisplatin is produced by apoptosis. The present study demonstrated that Epo inhibited apoptosis mediated by cisplatin. Caspases are key elements in apoptosis, and it was demonstrated that Epo significantly reduced the activity of caspase-3, which was in agreement with the evidence that multidrug-resistance was correlated with a reduced caspase-3 activity in cervical cancer cells (21).

The JAK/STAT pathway is crucial for the transduction of signals from activated EpoR. In particular, the JAK2/STAT5 signalling has been identified as acting as a promoter of malignant cell activities $(22,23)$. However, other members of the STAT transcription factors family, including STAT3, have been implicated in cancer progression (24). In a former study, we observed that EpoR was an upstream regulator of STAT3 (9). Accordingly, the present study demonstrated that Epo activated a JAK2/STAT3 cascade that mediated cytoprotection against cisplatin-induced cell death. JAK2 is a master kinase initiating cancer-associated cascades from growth factor receptors. Thus, inhibition of JAK2 has been considered as a promising target for cancer treatment. Notably, a recent study demonstrated that by combining mathematical and experimental tools it was possible to find differences between signalling axes activated in normal and tumour cells (19). Using this approach, the authors observed that inhibition of JAK2 in combination with Epo treatment had a stronger effect in lung cancer cells compared with erythroid progenitor cells, suggesting that blocking this pathway may inhibit the cytoprotective effects of Epo during chemotherapy. Activation of EpoR in erythroid cells normally stimulates phosphorylation and the nuclear translocation of STAT5. The present study observed that in cervical cancer cells EpoR activation also triggered the phosphorylation and transcriptional activity of STAT3. In our model, inhibition of STAT3 phosphorylation reversed the cytoprotective effect of Epo, indicating that STAT3 is a regulator of cell death inhibition. In agreement with the present study, Wei et al (25) reported that Epo saved neuronal cells from apoptosis following brain injury by activating a JAK2/STAT3 cascade.

STAT3 is a transcriptional activator of genes involved in cell survival. In fact, STAT3 activation protects breast cancer cells from apoptosis by inducing the expression of survivin (26). The present study demonstrated that following stimulation with Epo, STAT3 induced the expression and activation of survivin producing a fully active, anti-apoptotic molecule. The participation of survivin in cell protection against cisplatin toxicity has previously been reported in a non-small cell lung cancer model (27). It is well known that survivin is overexpressed in the majority of types of cancer (28), including cervical cancer (29). For this reason, survivin has been considered as a promising target for designing innovative cancer therapies. One of these strategies has been the development of molecules to inhibit survivin gene transcription. In the present study, the inhibitor YM155 was used, which blocks survivin gene 
transcription and has been demonstrated to overturn cisplatin resistance in head and neck cancer. Although it was observed that the transcriptional inhibition of survivin had a significant effect on the cytoprotective effect of Epo, it was not able to completely reverse the Epo-associated cisplatin resistance in our model. Since STAT3 activates the transcription of a number of genes, it is probable that survivin acts simultaneously with other factors. Thus, a more feasible approach to inhibit Epo-induced cytoprotection may be to target STAT3 itself.

Constitutive activity of STAT3 has been observed in numerous types of cancer. In cervical cancer patients, phosphorylation of STAT3 was revealed to be correlated with lymph node metastasis and tumour size, thus STAT3 activation is considered a predictor of poor prognosis. Blocking STAT3 phosphorylation using the WP1066 inhibitor has been demonstrated to reduce the size of gastric tumours in mice (16) and to induce the death of renal cell carcinoma cells in vitro and in vivo. Furthermore, inhibition of STAT3 phosphorylation by WP1066 was revealed to induce the anoikis of cervical cancer cells. The present study demonstrated that pre-incubation with sub-lethal concentrations of WP1066 abrogated the cytoprotective effect of Epo and restored cisplatin sensitivity in cervical cancer cells. This observation was in agreement with a previous study revealing that WP1066 was able not only to reverse chemoresistance, but also to increase the toxic effect of cisplatin in cisplatin-resistant oral squamous cell carcinoma cells.

In conclusion, the results of the present study demonstrated that exogenous Epo stimulated signalling pathways regulated by STAT3 and altered the response to cisplatin, and indicated that blocking STAT3 activation may improve the chemotherapeutic efficacy of cisplatin in cervical cancer patients requiring Epo as a treatment for anaemia.

\section{Acknowledgements}

The authors would like to thank Dr Miguel Tapia Rodríguez and Dr Gerardo Arrellín Rosas for their excellent technical assistance.

\section{Funding}

The present study was supported by a grant fromPAPIIT,UNAM (grant no. IN204816). Scholarships from CONACyT (Mexico City, Mexico) were awarded to LGCB (reg. no. 449748), GVG (reg. no. 324651) and ICBF (reg. no. 393137).

\section{Availability of data and materials}

All data generated or analysed during this study are included in this published article.

\section{Authors' contributions}

MJVM determined the effect of Epo on cell lines, evaluated apoptosis and caspase- 3 activation, analysed and interpreted the data. LGCB performed the experiments of inhibition of EpoR and JAK2, and analysed the expression of JAK2, STAT3, and survivin. ICBF performed in vitro assays using MY155 and WP1066 and analysed the data. CA evaluated the effect of
Epo in the xenograft model, analysed and interpreted the data. GVG evaluated the effect of WP1066 in the xenograft model, analysed the data and participated in the writing of the manuscript. LRZ conceived the idea, designed the experiments, analysed and interpreted the data, and wrote the manuscript. All authors read and approved the manuscript and agree to be accountable for all aspects of the research in ensuring that the accuracy or integrity of any part of the work are appropriately investigated and resolved.

\section{Ethics approval and consent to participate}

Experimental procedures were carried out in accordance with the Guidelines for the Care and Use of Laboratory Animals of the Panamerican University (Mexico City, Mexico) which follow internationally recognized guidelines on animal welfare.

\section{Patient consent for publication}

Not applicable.

\section{Competing interests}

The authors declare that they have no competing interests.

\section{References}

1. Weiss G and Goodnough LT: Anemia of chronic disease. N Engl J Med 352: 1011-1023, 2005.

2. Steegmann JL, Sanchez Torres JM, Colomer R, Vaz A, Lopez J, Jalon I, Provencio M, Gonzalez-Martin A and Perez M: Prevalence and management of anaemia in patients with non-myeloid cancer undergoing systemic therapy: A Spanish survey. Clin Transl Oncol 15: 477-483, 2013.

3. Szenajch J, Wcislo G, Jeong JY, Szczylik C and Feldman L: The role of erythropoietin and its receptor in growth, survival and therapeutic response of human tumor cells from clinic to bench-A critical review. Biochim Biophys Acta 1806: 82-95, 2010.

4. Marti HH: Erythropoietin and the hypoxic brain. J Exp Biol 207: 3233-3242, 2004.

5. Wang Y, Zhang H, Liu Y, Li P, Cao Z and Cao Y: Erythropoietin (EPO) protects against high glucose-induced apoptosis in retinal ganglional cells. Cell Biochem Biophys 71: 749-755, 2015.

6. Pezeshki Z, Nematbakhsh M, Mazaheri S, Eshraghi-Jazi F, Talebi A, Nasri H, Safari T, Mansouri A and Ashrafi F: Estrogen abolishes protective effect of erythropoietin against cisplatin-induced nephrotoxicity in ovariectomized rats. SRN Oncol 2012: 890310, 2012.

7. Ferlay J, Soerjomataram I, Dikshit R, Eser S, Mathers C, Rebelo M, Parkin DM, Forman D and Bray F: Cancer incidence and mortality worldwide: Sources, methods and major patterns in GLOBOCAN 2012. Int J Cancer 136: E359-E386, 2015.

8. Candelaria M, Cetina L and Dueñas-Gonzalez A: Anemia in cervical cancer patients. Implications for iron supplementation therapy. Med Oncol 22: 161-168, 2005.

9. Lopez TV, Lappin TR, Maxwell P, Shi Z, Lopez-Marure R, Aguilar C and Rocha-Zavaleta L: Autocrine/paracrine erythropoietin signalling promotes JAK/STAT-dependent proliferation of human cervical cancer cells. Int J Cancer 129: 2566-2576, 2011.

10. Aguilar C, Aguilar C, Lopez-Marure R, Jimenez-Sanchez A and Rocha-Zavaleta L: Co-stimulation with stem cell factor and erythropoietin enhances migration of c-kit expressing cervical cancer cells through the sustained activation of ERK1/2. Mol Med Rep 9: 1895-1902, 2014.

11. Hamadmad SN and Hohl RJ: Lovastatin suppresses erythropoietin receptor surface expression through dual inhibition of glycosylation and geranylgeranylation. Biochem Pharmacol 74: 590-600, 2007. 
12. Fukada T, Hibi M, Yamanaka Y, Takahashi-Tezuka M, Fujitani Y, Yamaguchi T, Nakajima K and Hirano T: Two signals are necessary for cell proliferation induced by a cytokine receptor gp130: Involvement of STAT3 in anti-apoptosis. Immunity 5: 449-460, 1996.

13. Gu L, Chiang KY, Zhu N, Findley HW and Zhou M: Contribution of STAT3 to the activation of survivin by GM-CSF in CD34 ${ }^{+}$cell lines. Exp Hematol 35: 957-966, 2007.

14. O'Connor DS, Grossman D, Plescia J, Li F, Zhang H, Villa A, Tognin S, Marchisio PC and Altieri DC: Regulation of apoptosis at cell division by $\mathrm{p} 34^{\mathrm{cdc} 2}$ phosphorylation of survivin. Proc Natl Acad Sci USA 97: 13103-13107, 2000.

15. Wall NR, O'Connor DS, Plescia J, Pommier Y and Altieri DC: Suppression of survivin phosphorylation on Thr34 by flavopiridol enhances tumor cell apoptosis. Cancer Res 63: 230-235, 2003.

16. Judd LM, Menheniott TR, Ling H, Jackson CB, Howlett M, Kalantzis A, Priebe W and Giraud AS: Inhibition of the JAK2/STAT3 pathway reduces gastric cancer growth in vitro and in vivo. PLoS One 9: e95993, 2014.

17. Nakahara T,Kita A, Yamanaka K, Mori M,Amino N, Takeuchi M, Tominaga F, Hatakeyama S, Kinoyama I, Matsuhisa A, et al: YM155, a novel small-molecule survivin suppressant, induces regression of established human hormone-refractory prostate tumor xenografts. Cancer Res 67: 8014-8021, 2007.

18. Alural B, Ayyildiz ZO, Tufekci KU, Genc S and Genc K: Erythropoietin promotes glioblastoma via miRNA-451 suppression. Vitam Horm 105: 249-271, 2017.

19. Merkle R, Steiert B, Salopiata F, Depner S, Raue A, Iwamoto N Schelker M, Hass H, Wasch M, Bohm ME, et al: Identification of cell type-specific differences in erythropoietin receptor signalling in primary erythroid and lung cancer cells. PLoS Comput Biol 12: e1005049, 2016.

20. Zhu H, Luo H, Zhang W, Shen Z, Hu X and Zhu X: Molecular mechanisms of cisplatin resistance in cervical cancer. Drug Des Devel Ther 10: 1885-1895, 2016.
21. Ding Z, Yang X, Pater A and Tang SC: Resistance to apoptosis is correlated with the reduced caspase- 3 activation and enhanced expression of antiapoptotic proteins in human cervical multidrug-resistant cells. Biochem Biophys Res Commun 270: 415-420, 2000

22. Lai SY, Childs EE, Xi S, Coppelli FM, Gooding WE, Wells A Ferris RL and Grandis JR: Erythropoietin-mediated activation of JAK-STAT signaling contributes to cellular invasion in head and neck squamous cell carcinoma. Oncogene 24: 4442-4449, 2005.

23. Shi Z, Hodges VM, Dunlop EA, Percy MJ, Maxwell AP, El-Tanani $M$ and Lappin TR: Erythropoietin-induced activation of the JAK2/STAT5, PI3K/Akt, and Ras/ERK pathways promotes malignant cell behaviour in a modified breast cancer cell line. Mol Cancer Res 8: 615-626, 2010.

24. Yu H, Lee H, Herrmann A, Buettner R and Jove R: Revisiting STAT3 signalling in cancer: New and unexpected biological functions. Nat Rev Cancer 14: 736-746, 2014

25. Wei S, Luo C, Yu S, Gao J, Liu C, Wei Z, Zhang Z, Wei L and Yi B: Erythropoietin ameliorates early brain injury after subarachnoid haemorrage by modulating microglia polarization via the EPOR/JAK2-STAT3 pathway. Exp Cell Res 361: 342-352, 2017.

26. Gritsko T, Williams A, Turkson J, Kaneko S, Bowman T, Huang M, Nam S, Eweis I, Diaz N, Sullivan D, et al: Persistent activation of STAT3 signaling induces survivin gene expression and confers resistance to apoptosis in human breast cancer cells. Clin Cancer Res 12: 11-19, 2006.

27. Hu W, Jin P and Liu W: Periostin contributes to cisplatin resistance in human non-small cell lung cancer A549 cells via activation of Stat 3 and Akt and upregulation on survivin. Cell Physiol Biochem 38: 1199-1208, 2016.

28. Altieri DC: Survivin, cancer networks and pathway-directed drug discovery. Nat Rev Cancer 8: 61-70, 2008.

29. Fan Y and Chen J: Clinicopathological significance of survivin expression in patients with cervical cancer: A systematic meta-analysis. Bioengineered 8: 511-523,2017. 